Relations industrielles

Industrial Relations

\title{
Les comités de santé et de sécurité du travail: tables de concertation ou de négociation?
}

\section{Kenneth George}

Volume 40, numéro 3, 1985

URI : https://id.erudit.org/iderudit/050158ar

DOI : https://doi.org/10.7202/050158ar

Aller au sommaire du numéro

Éditeur(s)

Département des relations industrielles de l'Université Laval

ISSN

0034-379X (imprimé)

1703-8138 (numérique)

Découvrir la revue

Citer cet article

George, K. (1985). Les comités de santé et de sécurité du travail: tables de concertation ou de négociation? Relations industrielles / Industrial Relations, 40(3), 512-528. https://doi.org/10.7202/050158ar
Résumé de l'article

Cet article cherche à caractériser l'expérience des comites de sante et de sécurité du travail par rapport à certains des objectifs importants à l'origine.
Tous droits réservés @ C Département des relations industrielles de l'Université Laval, 1985
Ce document est protégé par la loi sur le droit d'auteur. L'utilisation des services d'Érudit (y compris la reproduction) est assujettie à sa politique d'utilisation que vous pouvez consulter en ligne.

https://apropos.erudit.org/fr/usagers/politique-dutilisation/ 


\title{
Les comités de santé et de sécurité du travail
}

\section{Tables de concertation ou de négociation?}

\author{
Kenneth George
}

Cet article cherche à caractériser l'expérience des comités de santé et de sécurité du travail par rapport à certains des objectifs importants à l'origine.

La loi québécoise sur la santé et la sécurité du travail prévoit la création, dans certaines entreprises, d'un comité de santé et de sécurité du travail. En juillet 1984, 500 comités étaient formés dans des entreprises de plus de vingt travailleurs dans neuf secteurs d'activités économiques ${ }^{1}$.

Même si cette expérience est encore très jeune ${ }^{2}$, on peut, à partir de certaines données, commencer à déceler des tendances et formuler des hypothèses de recherche quant à la dynamique de ces comités. En effet, quelques acteurs ont commencé à caractériser et évaluer ces comités; des analystes ont publié des études qui, même si elles ne portent pas spécifiquement sur les comités de santé et de sécurité du travail, peuvent fournir des indications sur ce que l'avenir peut réserver à ces comités. L'objectif de cette recherche est de faire une revue de divers documents et études desquels on pourrait dégager les tendances qui semblent $s$ 'affirmer ou pourraient se développer dans les comités de santé et de sécurité du travail.

Ce ne sont pas tous les aspects de cette expérience qui retiendront l'attention. La préoccupation première sera de caractériser l'expérience des

* GEORGE, Kenneth, Commission consultative sur le travail, Montréal.

** Pour leurs commentaires sur une première version de ce texte, je veux remercier Gilles Laflamme (U. Laval), Florian Ouellet (U. de Montréal), Richard Goyette (local 62 - CPQMC) et Diane Chevalier, Laurent Gratton, François Paquin et Diane Rodier (CSST: Division Associations sectorielles paritaires).

1 Jean-Louis BERTRAND, «L'implantation des comités paritaires d'établissement de SST (loi 17)", Normes du travail: impacts sur la gestion des ressources humaines et sur les rapports collectifs de travail, rapport du $\mathrm{XV}^{\mathrm{e}}$ colloque des relations industrielles de l'Université de Montréal, 1984, pp. 207-210.

2 Le règlement est entré en vigueur le 22 octobre 1983 pour les entreprises de certains secteurs industriels. 
comités de santé et de sécurité du travail par rapport à certains des objectifs importants visés à l'origine; plus précisément, il s'agira de voir si, via ces comités d'entreprise, on réussit à sortir les enjeux de la santé et de la sécurité du travail du champ de la négociation pour en faire des objets de concertation.

Dans la première partie de ce texte, on rappellera les perspectives à la base de la loi. Dans la seconde partie, on évaluera si, dans les entreprises où existe une organisation syndicale, l'expérience des comités de santé et de sécurité du travail correspond à ces perspectives. Dans la troisième partie, on s'arrêtera à la situation dans les entreprises non syndiquées.

\section{LES PERSPECTIVES ORIGINALES}

Une des perspectives importantes - révolutionnaires, selon certains du livre blanc ${ }^{3}$ et de la loi était d'affirmer la responsabilité première des travailleurs et des employeurs face à la santé et la sécurité en situation de travail. Certes, via la négociation des conventions collectives, syndicats et employeurs traitaient déjà de ces «conditions de travail»"; mais nombre de groupes de travailleurs parvenaient difficilement à faire reconnaître la légitimité de leur intervention et les mécanismes d'intervention, ceux de la négociation collective, semblaient problématiques à plusieurs.

Ce que faisait la loi, c'était d'abord de légitimer l'intervention des travailleurs en santé et sécurité du travail. Comme l'explique Gratton, la participation des travailleurs ferait en sorte que

«les objectifs économiques qui sont les déterminants premiers du comportement patronal ne seraient pas les seuls critères de reconnaissance des problèmes de santé et de sécurité des travailleursm. ${ }^{5}$

En plus de permettre une compréhension plus juste des problèmes, la participation des travailleurs était nécessaire pour que le mécanisme d'action soit perçu comme crédible par les travailleurs. Dans ces conditions,

3 Gouvernement du Québec, Santé et sécurité au travail: politique québécoise de la santé et de la sécurité du travail, Québec, Éditeur officiel du Québec, 1978, 289 pages; voir, en particulier, pp. 199-219.

4 Voir, à ce sujet, Chantal BRISSON, L'utilisation de la convention collective en tant qu'outil d'intervention dans le domaine de la santé au travail, Québec, Université Laval, Département des relations industrielles, collection Instruments de travail, 1982, 166 pp. René BOULARD et Michel MARCHAND «La loi sur la santé et la sécurité du travail et les conventions collectives», Relations industrielles, vol. 38, no 4, pp. 847-862.

5 Laurent GRATTON, «Une association sectorielle paritaire: solution de rechange en matière de santé et de sécurité du travail», Le Devoir, 29 août 1984, p. 9. 
l'action en santé et sécurité du travail serait plus efficace i.e. «rentable pour l'entreprise, l'employeur et, surtout et avant tout, pour la santé et la sécurité des travailleurs ${ }^{6}$.

En plus, pour opérationnaliser ce principe, la loi créait des mécanismes de participation des travailleurs - participation décisionnelle - à la définition de conditions saines et sécuritaires de travail; ces comités de santé et de sécurité du travail devaient exister parallèlement aux mécanismes habituels de la négociation collective.

En d'autres mots, si on voulait que toutes les parties s'engagent en santé et sécurité du travail, on ne voulait pas que cet engagement s'exprime dans une dynamique de négociation, de rapport de force, on ne voulait pas que la santé et la sécurité du travail ne soient que d'autres enjeux dans le système conflictuel des relations du travail. Ce qui était espéré, c'est que les acteurs en viennent à considérer la santé et la sécurité du travail comme étant fondamentalement différentes des autres enjeux et que, en conséquence, ils les abordent dans un modèle de relation différent du modèle traditionnel de lutte de pouvoir. Plutôt que la négociation entre acteurs sur ces enjeux, on privilégiait la concertation sur ces sujets. La santé et la sécurité du travail devaient cesser d'être des enjeux à débattre et devenir des valeurs qui appellent l'adhésion de tous.

La thèse selon laquelle la santé et la sécurité du travail devraient être exclues du champ de la négociation collective a été développée, entre autres, par Robert Sauvé7. Même si théoriquement tout se négocie, Sauvé fait valoir que la négociation collective est toute orientée en fonction d'enjeux reliés aux salaires - cela a un fondement historique évident - et elle est donc inappropriée pour l'étude des problèmes de santé et de sécurité du travail; d'ailleurs, précise-t-il, l'expérience enseigne que, quand on négocie la santé et la sécurité du travail, on a plutôt tendance à succomber à la tentation d'intégrer ces enjeux dans un marchandage où les questions pécuniaires l'emportent facilement.

Florian Ouellet ${ }^{8}$ fait une analyse semblable. C'est dans le cadre de la négociation collective que syndicats et employeurs ont imaginé les «primes de danger» - qui existent d'ailleurs toujours dans certaines conventions

6 Ibid.

7 Robert SAUVE, «Changements en matière de santé et de sécurité au Québec». La version française de ce texte n'a pas été publiée. Une traduction anglaise paraît sous le titre: «Regulation through Legislation: Perspectives for Health and Safety in Québec», Are Health and Safety Negotiable? Montréal, McGill University, Industrial Relations Centre, 26th Annual Conference, 1978, pp. 32-47.

8 FLorian OUELLET, La santé et la sécurité au travail: pour une action sur les lieux de travail, Montréal, Institut de recherche appliquée sur le travail, 1979. 
collectives9. L'expérience enseigne aussi qu'en certaines circonstances, la santé et la sécurité du travail ne sont même pas des enjeux de négociation mais tout simplement un moyen de pression syndicale: «lorsqu'on a obtenu ce que l'on demande, on relâche les pratiques de sécurité» ${ }^{10}$. Les employeurs jouant le même jeu, les conditions de travail ne changent pas. Bref, comme Sauvé, Ouellet doute que la négociation collective puisse véritablement assurer des conditions de travail saines et sécuritaires, à moins de changements importants dans les attitudes des acteurs.

Quoique pour des raisons quelque peu différentes, Gibbs ${ }^{11}$ en arrive à la même conclusion. La négociation des conditions de travail n'oriente pas vers des solutions à des problèmes mais plutôt vers des compromis entre des intérêts; il ne faudrait pas être surpris alors que ces compromis soient peu pertinents aux problèmes quotidiens de santé et de sécurité du travail. Plus grave encore est le fait que, pour résoudre nombre de problèmes de santé et de sécurité du travail, une recherche continue, ordonnée et quelquefois longue est nécessaire; or, toutes les nuances d'un programme ou d'une méthodologie de recherche ne sauraient s'exprimer dans une clause de convention collective.

Pour Sauvé et Gibbs ${ }^{12}$, compte tenu que la négociation collective et la lutte de pouvoir ne peuvent pas assurer des conditions saines et sécuritaires de travail, il faut développer de nouveaux mécanismes où les acteurs puissent assumer leurs responsabilités, des mécanismes d'où seront absentes les luttes de pouvoir que suppose la négociation collective, des mécanismes de concertation comme les comités de santé et de sécurité du travail.

Il ne saurait être question cependant que ces nouvelles structures ne soient qu'un autre lieu d'affrontements entre les parties; à cette fin,

"cette structure doit être complètement autonome en ce sens qu'elle fonctionne en dehors des préoccupations du marchandage économique et repose sur des personnes qui ont une responsabilité limitée à la prévention... (À ces conditions, les parties discuteront) dans un climat de confiance a l'abri du contexte traditionnel d'affrontement des relations de travail». ${ }^{13}$

9 À ce sujet, voir Diane CHEVALIER, «Les clauses d'indemnité pour travail dangereux, salissant ou autres conditions et la rémunération au rendement dans les conventions collectives signées au cours de la période 1981-1984», CSST, Division: Associations sectorielles paritaires, $1984,16 \mathrm{pp}$., étude non publiée; des données de cette étude paraissent sous le titre «Primes versées aux travailleurs», CSST '85, mai 1985, p. 3.

10 FLorian OUELLET, op. cit., p. 12.

11 Graham William GIBBS, «Health Hazards: Confrontation Issues on the Job», Are Health and Safety Negotiable?, supra, pp. 66-79.

12 Ouellet propose plutôt que, s'ils en viennent à reconnaître le caractère spécial de ces enjeux et à ne plus les marchander, les syndiqués pourraient, via la négociation collective, obtenir de très bonnes conditions de santé et de sécurité du travail que l'état pourrait ensuite imposer aux entreprises où les travailleurs sont moins bien organisés.

13 Robert SAUVÉ, op. cit., p. 17. 
Deux aspects opérationnels et une dimension psychologique devraient donc caractériser les comités et faire en sorte qu'ils soient des tables de concertation. Le premier aspect opérationnel a trait à la composition du comité: ne devraient pas y siéger des travailleurs ou des représentants patronaux qui s'occupent d'autres questions du champ des relations patronales-ouvrières. Le second aspect opérationnel a trait au mode d'étude des questions: les problèmes soumis à l'attention des membres du comité doivent être étudiés à leur mérite et non comme monnaie d'échange face à un autre enjeu. Enfin, dernière caractéristique, les membres du comité seront amenés, dans ces conditions, à travailler dans un climat de confiance i.e. sans se préoccuper de créer un précédent, sans cacher des renseignements, sans être obligés de prévoir une stratégie de repli. Ce sont là quelques-uns des éléments qui feront qu'on ne négociera pas la santé et la sécurité des travailleurs, on travaillera plutôt «de concert» pour régler les problèmes.

Comme on le voit, par rapport à la situation vécue depuis si longtemps, les nouvelles perspectives en santé et sécurité du travail posaient un défi à l'ensemble des acteurs. Affirmer la légitimité de l'intervention des travailleurs, créer des mécanismes d'intervention parallèles à ceux qui existaient, favoriser un changement dans les attitudes des parties, modifier les conceptions traditionnelles de la santé et la sécurité du travail, bref amener les parties à ne plus négocier un enjeu pour plutôt se concerter sur un objet de valeur, c'était là entraîner les acteurs sur des voies qui, on s'en doute bien, n'allaient pas sans poser de problème.

\section{LE CAS DE L'ENTREPRISE SYNDIQUÉE}

\section{La perspective patronale}

C'est le directeur général de l'Association des mines de métaux du Québec, L. Gonzague Langlois qui a le mieux résumé, récemment, la position patronale maintes fois développée par rapport à la santé et la sécurité du travail: l'action en ces domaines n'a de sens que si elle est exclusivement sous le contrôle patronal, condition nécessaire pour assurer le développement de l'entreprise.

Certes, comme le souligne Langlois, aucun employeur ne s'opposerait à «la collaboration sincère et efficace de la part des travailleurs, même au sein d'organismes paritaires»; mais cette collaboration doit être soumise «au rôle prioritaire de la gérance dans l'administration de l'entreprise même au niveau (sic) de la santé-sécurité du travail» ${ }^{14}$. Langlois précise que cette

14 L. Gonzague LANGLOIS, «Les associations sectorielles paritaires: souhaitables mais difficiles à réaliser», Le Devoir, 22 octobre 1984, p. 11. 
collaboration devrait mener, non pas à une action efficace pour la santé et la sécurité des travailleurs, mais à une amélioration de la productivité de l'entreprise, comme l'avait déjà signifié le président de Bell Canada que cite Langlois: «Le premier facteur de productivité dans l'entreprise est la santésécurité du travail» ${ }^{15}$.

Ces propos ne devraient pas surprendre. Ils ne font que refléter le fait qu'en Amérique du Nord, il n'y a pas de tradition d'ouverture patronale à quelque forme que ce soit de partage du pouvoir dans l'entreprise - ce qui est le véritable enjeu. Thomas Kochan rend bien compte de cette situation. Analysant l'expérience américaine de tables de rencontres syndicalespatronales, qu'il y soit question de santé et de sécurité du travail, de qualité de vie au travail ou de changements technologiques, Kochan explique que dans tous les cas, quand il s'agit d'aller au-delà du processus de la négociation collective, c'est sans enthousiasme, obligés, que les employeurs s'engagent dans ce type d'expérience qui met toujours en cause le partage du pouvoir dans l'entreprise:

«The management community opposes the idea for the obvious reason that it requires sharing decision-making authority beyond the scope of collective bargaining. Consequently, management resists this type of power sharing as it resists most other forms of structural redistribution of power in the organization». ${ }^{16}$

Dans ce contexte, il n'est pas surprenant que, appelé récemment à présenter l'évaluation faite par des employeurs, ou leurs représentants, qui siègent depuis un certain temps dans des comités de santé et de sécurité du travail, un analyste du Conseil du patronat du Québec, Pierre Duguay, n'a pu faire part d'aucun actif au bilan de ces comités. Cela correspond au fait qu'il n'y a eu aucun changement dans les attitudes des employeurs qui, comme Duguay le rappelle, «lors du débat sur la Loi $17, \ldots$ se sont opposés au caractère décisionnel du comité de santé et de sécurité du travail»» ${ }^{17}$, bref, au partage du pouvoir dans l'entreprise.

Sur la question de la participation des travailleurs à la prise de décision par le biais d'un comité de santé et de sécurité du travail, «les employeurs ne sont pas à l'aise». Ce sentiment s'explique du fait que

15 L. Gonzague LANGLOIS, "Une association de services techniques: solution de rechange à l'intervention gouvernementale dans le secteur minier", Le Devoir, 17 juillet 1984, p. 7.

16 Thomas KOCHAN, Collective Bargaining and Industrial Relations, Homewood, Ill., Richard D. Irwin Inc., 1980, p. 437.

17 Pierre DUGUAY, «L'implantation des comités paritaires d'établissement de SST (loi 17)», Normes du travail:..., supra, p. 212. 
«c'est l'employeur qui, dans la gestion de son entreprise, est responsable de protéger la santé et la sécurité de ses travailleurs. L'article 51 de la Loi 17 le précise clairement». ${ }^{18}$

Ainsi, puisque l'employeur est seul à assumer cette responsabilité, il n'y a aucun sens à faire partager son pouvoir de décision.

Cette responsabilité particulière de l'employeur explique aussi que pour lui, le comité de santé et de sécurité du travail ne saurait être considéré autrement que comme un outil de gestion de la santé et la sécurité du travail,

«un moyen parmi d'autres mais pas le seul et... dans certains cas, ce n'est de toute évidence pas le bon moyen d'améliorer la santé et la sécurité du travail». ${ }^{19}$

Bref, aucune valeur n'est reconnue à la participation des travailleurs en tant que telle; qui plus est, la référence à la responsabilité légale exclusive de l'employeur peut même laisser croire que non seulement la participation des travailleurs serait illégitime mais même, à la limite, elle constituerait un obstacle à l'exercice de la responsabilité patronale.

L'évaluation patronale porte aussi sur le type de relations qui se développent entre les membres des comités, la dynamique des comités: le comité «devient un lieu d'affrontement comme le sont les tables de négociation» et cela obligera l'employeur à faire appel, «comme pour les relations du travail», à des spécialistes nouveaux. Selon Duguay, ce qui favorise cette association entre les comités de santé et de sécurité du travail et les tables traditionnelles de négociation, c'est que les choses se déroulent de la même façon aux deux tables, une des parties est en position

«de demander et de ne rien débourser alors que l'autre partie reçoit des demandes et porte seule le poids financier des décisions du comité paritaire». ${ }^{20}$

Les propos de Langlois et de Duguay ne manifestent aucunement une quelconque reconnaissance patronale de la légitimité d'une intervention décisionnelle des travailleurs quand il s'agit de santé et de sécurité du travail. Ils indiquent aussi que l'employeur qui siège au comité de santé et de sécurité du travail se perçoit en situation de négociation, son schème de référence est celui de la négociation collective, c'est sur ce modèle qu'il vit la dynamique patronale-syndicale. Quant aux problèmes étudiés ou aux demandes formulées en comité, l'employeur les aborde de la même façon qu'il aborde un projet de convention collective déposé par un syndicat c'està-dire à travers le prisme traditionnel de son droit de gérance et de sa capacité de payer.

$\begin{array}{ll}18 & \text { Id., p. } 213 . \\ 19 & \text { Id., p. } 212 . \\ 20 & \text { Ibid. }\end{array}$


Somme toute, on aurait tort d'affirmer que les attitutdes patronales dans les comités de santé et de sécurité du travail correspondent aux perspectives nouvelles. On aurait tort de croire aussi que ce nouveau mécanisme est en voie de provoquer un changement d'attitudes. Il semble bien que la santé et la sécurité du travail resteront pendant un certain temps encore des enjeux de négociation plutôt que des objets de concertation.

\section{La perspective syndicale}

En ce qui regarde les travailleurs syndiqués, la question de la légitimité d'une «participation décisionnelle» dans la détermination des conditions de santé et de sécurité sur les lieux de travail ne se pose pas. Très simplement, comme le suggère Robert Bouchard, directeur de la santé et de la sécurité du travail à la Fédération des travailleurs du Québec, il y a conflit de pouvoir dans l'entreprise, conflit résultant de la coexistence légitime de «deux droits de propriété: celui de l'employeur sur le matériel et celui du travailleur sur sa personne» ${ }^{21}$. Ces deux droits expliquent que le pouvoir de l'employeur ne soit pas absolu, qu'il doive être partagé: le droit des travailleurs sur leur personne justifie que les travailleurs aient une participation décisionnelle à la détermination des conditions saines et sécuritaires de travail.

Cette participation passe par deux approches. La négociation collective des conditions de travail est la forme traditionnelle de participation, la forme qui, selon le mouvement syndical, a fait ses preuves, la forme d'action qui a permis le développement du mouvement syndical. La seconde forme, celle que propose la loi sur la santé et la sécurité du travail, celle que le mouvement syndical utilise par moments, c'est le comité conjoint, le comité d'entreprise, toutes les formes qu'on associe habituellement à la concertation.

Le mouvement syndical en Amérique du Nord a toujours été hésitant, mal à l'aise, par rapport à cette seconde forme d'action: il a toujours eu une prédisposition pour la négociation collective comme forme privilégiée d'action. Pour Kochan, la crainte de la «récupération» et le désir de ne pas limiter les moyens d'action expliqueraient cette attitude syndicale:

«The labor movement traditionnally has been cool to these participation systems because of its fear of being co-opted into participating in unpopular decisions. Instead, the dominant philosophy guiding the American labor movement has been, and continues to be, to endorse the formal collective bargaining process but to refrain form expanding the scope of union involvement by entering into a formal partership with management». ${ }^{22}$

21 Robert BOUCHARD, «L'implantation des comités paritaires d'établissement de SST (loi 17)», Normes du travail:..., supra, p. 210.

22 Thomas KOCHAN, op. cit., p. 439. 
Le mouvement syndical maintiendra-t-il cette attitude générale par rapport à la santé et la sécurité du travail? À la lumière de trois attitudes particulières du mouvement syndical, force est de conclure que, si l'on veut bien exploiter le potentiel que peuvent représenter les comités de santé et de sécurité du travail, il ne saurait être question de ne pas considérer la santé et la sécurité du travail comme des enjeux de négociation, d'exclure ces enjeux du champ de la négociation collective, de laisser se développer de façon autonome des mécanismes de travail indépendants des structures d'action traditionnelle ou parallèles à elles, bref de miner ou de sembler miner de quelque façon que ce soit le processus de la négociation collective. Cette conclusion se dégage des attitudes adoptées par les syndicats en ce qui a trait a) au choix des représentants des travailleurs au comité de santé et de sécurité du travail, b) à la relation à établir dans ces comités avec les travailleurs non syndiqués, dans les entreprises où une partie seulement des travailleurs est syndiquée et c) à la formation donnée par les centrales syndicales aux travailleurs membres des comités.

Ce n'est pas n'importe qui qui peut agir comme représentant des travailleurs au comité de santé et de sécurité du travail: selon Bouchard ${ }^{23}$, les comités administratifs des syndicats locaux s'assurent que les délégués syndicaux soient de véritables délégués, détenant des mandats du syndicat, ayant à tout le moins, quand ils ne sont pas des membres du comité administratif, à faire rapport à une structure syndicale. D'aucune façon, le représentant des travailleurs ne saurait-il être ou paraitre sans lien formel avec les leaders syndicaux, autonome, non intégré à l'ensemble de l'action syndicale.

Contrairement à un des éléments opérationnels définis plus haut et qui devrait donner forme aux nouvelles perspectives, cette attitude des syndicats traduit la conception traditionnelle à l'effet que la santé et la sécurité du travail sont des enjeux comme les autres, des objectifs qu'on doit atteindre via les structures et processus habituels; ce n'est pas parce qu'un mécanisme particulier existe ou qu'un représentant des travailleurs y est spécialement affecté qu'il faut dissocier l'action en santé et sécurité du travail du reste du mouvement syndical.

À d'aucuns, cela semblera faire exprès pour que le travail au comité de santé et de sécurité soit, non pas un effort conjoint de tous pour la promotion de la santé et de la sécurité, mais plutôt une session organisée de négociation. Quand on se rappelle cependant l'ambivalence des organisations syndicales par rapport à ces nouveaux mécanismes, l'intégration des délégués syndicaux à la structure syndicale semble moins étrange; à tout le moins, elle s'explique.

23 Voir le résumé de la discussion en atelier in Normes du travail:..., supra, p. 216. 
Par ailleurs, cette attitude syndicale de ne pas trop «isoler» l'action en santé et sécurité du travail de l'organisation syndicale régulière se justifie, selon Bouchard, par deux autres considérations. D'abord, le syndicat, c'est la propriété des travailleurs, c'est eux qui le financent, c'est leur instrument; on ne devrait quand même pas s'offusquer du fait qu'ils s'en servent et ce , le plus efficacement possible - c'est le contraire qui serait anormal. En second lieu, on a beau prétendre que la santé et la sécurité du travail ne sont pas des enjeux de négociation mais, dès qu'on monnaye une demande en santé et sécurité du travail, on en fait en enjeu de négociation (d'ailleurs, face à une demande syndicale, il ne semble pas rare, selon Bouchard, que l'employeur rétorque que l'argent investi là ne pourra pas être utilisé pour d'autres enjeux) ${ }^{24}$.

Comme on le voit, dans le choix des délégués syndicaux au comité de santé et de sécurité du travail, les organisations syndicales font attention à ne pas dissocier le travail fait par rapport à ces enjeux de l'action régulière $\mathrm{du}$ mouvement syndical; les organisations syndicales sont réticentes à négliger la forme de participation décisionnelle à laquelle elles sont habituées, pour une autre, un peu neuve et jeune.

Cette attitude manifeste clairement aussi que les syndicats ne croient pas réaliste de considérer la santé et la sécurité du travail autrement que comme un bien monnayable. Certes, tout le monde est pour la vertu! Cette vertu passe cependant par le biais du profit, du droit de gérance et, dans ce contexte, les syndicats laissent entendre qu'on se tromperait en croyant que le travail dans des conditions saines et sécuritaires est une valeur universelle à laquelle adhèrent spontanément les employeurs et non pas un objectif à atteindre par la mobilisation des forces, le rapport de forces.

La seconde attitude, aussi notée par Bouchard, a trait à la participation des non-syndiqués aux travaux du comité de santé et de sécurité du travail, dans une entreprise où une partie seulement des travailleurs est syndiquée. Dans ce cas, constate Bouchard, malgré le règlement qui est très clair à ce sujet, les syndiqués ne veulent pas que les non-syndiqués siègent avec eux au comité25.

24 Le juriste André Côté tient des propos semblables: "Si on prend l'exemple des programmes de prévention en matière de santé et de sécurité au travail, il m'apparaît évident que leur mise en application, leur révision périodique et leur opération seront génératrices de coûts pour les employeurs, ce qui réduira d'autant leur marge de manoeuvre pour la négociation d'autres conditions de travail». Côté établit cette relation pour tous les programmes «sociaux» que, par des lois normatives, l'état impose aux entreprises (programme d'accès a l'égalité, programme d'embauche des personnes handicapées,...). «Impacts des normes sur les rapports collectifs», Normes du travail...., supra, p. 270.

25 Voir le résumé de la discussion en atelier in Normes du travail..., supra, p. 214. 
Bouchard ne cache pas que cette attitude crée une certaine tension avec "l'establishment» syndical qui avait cru que le travail avec les nonsyndiqués dans les comités de santé et de sécurité aurait été possible et, peutêtre même, profitable pour l'organisation syndicale i.e. il aurait pu amener les non-syndiqués à voir les mérites de l'organisation syndicale. Il est intéressant aussi de noter que, selon Bouchard, dans leur opposition aux visées des permanents syndicaux, les syndicats locaux jouissent de l'appui de nul autre que l'employeur pour qui les non-syndiqués n'ont jamais vraiment compté, ils ont toujours été comme une «quantité négligeable».

Au fond, cette attitude des syndicats locaux était prévisible. Elle est tout à fait cohérente avec l'attitude décrite plus haut et la perception syndicale de la santé et la sécurité du travail comme des enjeux qui se négocient toujours; or, quand on négocie, on n'a aucune raison de faire alliance, front commun, avec... des faibles, des non-syndiqués... dont l'apport à l'alliance syndicale est nul... et qui, de surcroît, aux moments chauds, n'auront pas la force de résister aux pressions patronales... bref, des «quantités négligeables». Dans la logique du rapport de forces, il est difficile de voir l'intérêt qu'il y aurait à faire front commun avec des faibles.

Il est une troisième attitude du mouvement syndical qui correspond aussi à la tendance exprimée à travers les deux attitudes dont Bouchard avait rendu compte. Cette attitude a trait à la formation donnée par les centrales syndicales aux travailleurs appelés à siéger dans les comités de santé et de sécurité du travail.

En étudiant les documents pédagogiques préparés par les centrales syndicales, on constate que, dans les sessions de formation, on ne se limite pas à enseigner comment faire un ordre du jour, analyser un poste de travail ou analyser un accident. Ce sont là des éléments essentiels qui occupent d'ailleurs une bonne partie du temps de formation. Mais ce n'est pas tout. $\mathrm{Si}$, tout au long du cours, on amène les travailleurs à prendre conscience de l'importance du comité comme lieu de détermination d'un programme de prévention, d'un programme de santé, etc., on n'oublie pas cependant de leur démontrer que leur force pour faire valoir leur point de vue est un peu faible, que le rapport des forces n'est pas tout à fait égal, que les travailleurs ne disposent pas au comité de santé et de sécurité des moyens habituels de pression que sont le grief ou la grève et qu'en conséquence, les représentants des travailleurs doivent toujours noter les demandes formulées à la table du comité et qui ne sont pas satisfaites pour que ces demandes soient intégrées au cahier des revendications lors de la prochaine négociation collective ${ }^{26}$.

26 Voir, en particulier, les documents pédagogiques préparés par les centrales pour leur "cours de base» en santé et sécurité du travail; les documents utilisés dans les cours plus techniques (analyse d'accidents, santé au travail,...) reprennent ces mêmes thèmes quoique, souvent, de façon moins explicite. 
Cette attitude ne devrait pas surprendre. Elle est tout à fait cohérente par rapport à celles définies plus haut. En effet, le mouvement syndical considérant que les questions étudiées au comité ne peuvent pas l'être à leur mérite, qu'elles sont toujours monnayables et qu'on ne pourra obtenir gain de cause sur certaines questions que par les voies traditionnelles, il n'est que dans l'ordre des choses que les organisations syndicales attirent l'attention de leurs représentants sur les limites du mécanisme nouveau et les moyens à prendre pour dépasser ces limites.

Que ce soit par rapport au choix des délégués syndicaux, à la relation à établir, s'il y a lieu, avec les travailleurs non syndiqués de l'entreprise ou à la formation donnée aux représentants des travailleurs qui siègent aux comités, les attitudes syndicales face aux comités de santé et de sécurité du travail correspondent à la logique de l'action traditionnelle du mouvement syndical. Non seulement la conception des enjeux de santé et de sécurité du travail ne change pas mais, en plus, les mécanismes nouveaux dont l'effet devrait être de provoquer, de favoriser un changement de conception et d'attitudes sont tout simplement redéfinis en fonction des "vérités» traditionnelles.

\section{LE CAS DE L'ENTREPRISE NON SYNDIQUÉE}

\section{La perspective patronale}

La vaste majorité des entreprises au Québec sont des PME non syndiquées où s'applique nombre de normes gouvernementales. Le professeur André Petit ${ }^{27}$ a essayé de comprendre la réaction patronale à ces normes dans les entreprises de ce type et, de son analyse, on pourrait dégager des hypothèses quant à l'attitude patronale vis-à-vis les comités de santé et de sécurité du travail.

Selon Petit, l'application d'une norme, comme l'établissement d'un comité de santé et de sécurité du travail, pose des problèmes psychologiques, financiers et organisationnels au chef d'entreprise. Il n'est pas toujours facile de comprendre un règlement, d'évaluer le coût d'application, de modifier les modes de gestion, de s'assurer que les cadres feront les adaptations nécessaires, de s'engager, bref, dans un processus de changement.

Et ce ne sont là que quelques-unes des difficultés. Si on ajoutait, comme le fait remarquer Petit, qu'au Canada, les gestionnaires ont des visions «simplistes et erronées» de ce qu'est la gestion des ressources humaines! On

27 André PETIT, «L'impact des normes du travail sur la gestion des ressources humaines», Normes du travail:..., supra, pp. 136-157. 
ne devrait alors pas être surpris de la conclusion à laquelle en arrive Petit: les lois sur les normes ont un impact très limité dans la réalité quotidienne des entreprises où elles devraient s'appliquer.

Compte tenu de cette caractérisation, quelle pourrait être l'attitude patronale, dans une entreprise non syndiquée, par rapport à un comité de santé et de sécurité du travail? par rapport à l'intervention des travailleurs? Si on extrapole à partir de la thèse d'André Petit, on pourrait formuler les hypothèses suivantes:

- l'employeur n'aura pas tendance à considérer la santé et la sécurité du travail comme des enjeux particuliers; à moins que des pressions extérieures ne s'exercent sur lui, il n'aura pas tendance à créer, dans son entreprise, une structure de participation des travailleurs pour faire face à ces enjeux;

- si les pressions sont assez fortes (visite d'un inspecteur, pressions de la CSST, du CLSC, du DSC,...), si, en particulier, un accident grave de travail survient dans l'entreprise, l'employeur acceptera la création d'un comité de santé et de sécurité du travail, qu'il ne saurait cependant considérer comme autonome par rapport à ses prérogatives;

- si les pressions extérieures diminuent, si le souvenir de l'accident qui a provoqué la création du comité de santé et de sécurité du travail se perd, les membres du comité ne seront plus convoqués en réunion; de toute façon, à la longue, ces réunions seront devenues des moments de rencontre où on ne sait plus trop ce qu'on y fait.

Bref, ce n'est pas de façon empressée que l'employeur cherchera à donner forme, dans la gestion de son entreprise, aux perspectives développées par la loi. S'il n'a pas l'occasion de nier formellement la légitimité d'une participation décisionnelle des travailleurs face à leur santé et leur sécurité, il ne saurait de lui-même créer un cadre d'action où les travailleurs pourraient s'exprimer. Cette attitude patronale est d'ailleurs d'autant plus facile à maintenir, malgré le discours politique et la présence de la CSST, qu'il n'y a pas dans l'entreprise de contre-pouvoir qui puisse provoquer des changements de la part de l'employeur. La santé et la sécurité du travail ne sont alors rien d'autre qu'un autre enjeu dans la relation entre l'employeur et ses employés, enjeu auquel l'employeur fera face de façon tout à fait traditionnelle.

\section{La perspective des travailleurs}

Comme on l'a fait dans le cas des employeurs, on peut tenter de caractériser la perspective des travailleurs non syndiqués par rapport aux 
comités de santé et de sécurité du travail à partir des études sur les attitudes de ces travailleurs face à d'autres droits, mécanismes et recours prévus dans diverses lois.

L'analyste Doris Armstrong a publié une étude sur l'application dans une entreprise des normes relatives aux congédiements, sans cause juste et suffisante, d'employés à qui la Loi sur les normes du travail offrait un recours. Elle a cherché à savoir si les employeurs à qui on avait ordonné de réintégrer des employés qui avaient porté une plainte de congédiement s'étaient conformés aux décisions, si, donc, les travailleurs avaient suffisamment de force pour exercer, appuyés en cela par l'État, leur droit à retrouver l'emploi qu'ils détenaient.

L'étude a révélé que, contrairement à ce à quoi on aurait pu s'attendre, seulement $54.2 \%$ de ces salariés ont été réintégrés dans leur emploi. C'est déjà là une première indication de la faiblesse des travailleurs non organisés qui ne réussissent même pas, appuyés par l'État, rappellons-le, à faire respecter leurs droits. Cette faiblesse apparaît encore plus grande quand on tient compte des faits suivants: parmi les personnes réintégrées, les deux tiers disent avoir été traitées injustement par leur employeur, un taux important démissionne «en raison du comportement de l'employeur» ${ }^{28}$ et $13.3 \%$ ont de nouveau été congédiées.

On ne saurait dégager de ces données que les travailleurs vont, du fait que la loi leur reconnaît certains droits, adopter une attitude "agressive» pour faire reconnaître ces droits. Par ailleurs, même si l'étude ne fournit pas de données sur le degré d'utilisation du recours prévu par la loi (quelle proportion de ceux qui seraient en droit de le faire déposent une plainte?), elle rend compte du fait que des travailleurs utilisent le mécanisme bureaucratique.

D'une deuxième étude, l'étude déjà présentée d'André Petit, on peut aussi dégager des conclusions semblables sur les attitudes des travailleurs par rapport aux droits définis dans les lois normatives. La thèse de Petit est que là où elles s'appliquent, les normes du travail ont un impact limité sur la gestion des ressources humaines, sur ce qui se passe en entreprise. Cette constatation laisse entrevoir que les travailleurs non syndiqués n'ont pas une forte propension à réagir quand l'employeur ne respecte pas trop les droits que la loi leur reconnaît.

Les études de Armstrong et de Petit démontrent toutes deux que, dans les entreprises non syndiquées, les travailleurs ont peu de prise sur la réalité. De surcroît, ces travailleurs ne semblent pas avoir les moyens d'affirmer

28 Doris ARMSTRONG, «L'efficacité de la réintégration ordonnée par l'arbitre», Le marché du travail, vol. 5, no 10,1984, p. 84. 
leurs droits. De fait, ils ne semblent avoir qu'un seul moyen d'action, si faible soit-il: le recours aux mécanismes bureaucratiques prévus dans la loi.

De ces études, on peut dégager les hypothèses suivantes quant aux attitudes des travailleurs non syndiqués face aux comités de santé et de sécurité du travail:

- les travailleurs n'auront pas tendance à considérer la santé et la sécurité du travail comme des enjeux à isoler de l'ensemble de ceux qui font la relation entre eux et leur employeur; ils n'auront pas tendance à adopter par rapport à la santé et la sécurité du travail des attitudes différentes de celles qu'ils ont habituellement vis-à-vis leur employeur;

- si un problème particulièrement grave se pose, plutôt que d'initier les démarches en vue de la création d'un comité de santé et de sécurité du travail, les travailleurs auront tendance à faire appel aux services appropriés de la CSST;

- si, à cause de pressions extérieures à l'entreprise ou pour d'autres raisons, un comité est créé, le dynamisme du comité dépendra essentiellement de l'apport d'intervenants extérieurs; vraisemblablement, le comité ne sera qu'un moyen d'action de l'employeur et aucunement un lieu efficace de participation des travailleurs.

Bref, la santé et la sécurité du travail sont toujours des éléments quelconques de la relation patronale-ouvrière, relation où le travailleur est en situation de subordination pour tout ce qui a trait au travail et à l'ensemble des conditions de travail, ce qui comprend, malgré la loi, les conditions de santé et de sécurité du travail.

\section{CONCLUSION}

Ce qui se dégage de cette analyse, c'est que, tant dans les entreprises syndiquées que dans les entreprises non syndiquées, la tendance nettement prédominante chez tous les acteurs est de continuer à traiter la santé et la sécurité du travail comme des enjeux de négociation ou d'une relation de pouvoir, bref, de façon traditionnelle; les acteurs font peu d'ouverture aux perspectives nouvelles.

Le reconnaissance de nouveaux droits et la création de nouvelles institutions où les acteurs prendraient en charge la santé et la sécurité du travail, de façon conjointe et concertée, devaient faire en sorte que ces enjeux ne soient plus du champ du politique ou du rapport de force; la valeur intégrative reconnue de la santé et de la sécurité du travail justifiait, entre 
autres, cet espoir. Or il arrive que si les mécanismes, les forums sont nouveaux, le comportement des acteurs n'est pas, lui, renouvelé. La santé et la sécurité du travail restent encore une affaire de pouvoir.

La pratique américaine annonçait d'ailleurs ces tendances, en particulier la «récupération» de tout nouveau mécanisme de concertation par le processus établi de la négociation collective. Les études démontrent en effet que les expériences de comités conjoints en santé et sécurité du travail ou par rapport à tout autre enjeu ne durent que dans la mesure où l'employeur et l'organisation des travailleurs parviennent à y trouver leur intérêt propre et où, à la fin, ces expériences sont intégrées dans le système formel de la négociation collective, système qui, comme le suggère Kochan, a déjà, lui, fait ses preuves:

«The institution of collective bargaining is too central to American industrial relations and deals too effectively with recurring economic problems and other issues where the goals of the parties are incompatible to expect that some new reform effort... can replace collective bargaining». ${ }^{29}$

Par rapport à la question de savoir si, dans l'avenir, on parviendra à faire de la santé et la sécurité du travail non des enjeux de négociation mais des objets de concertation, il semble qu'il faille constater qu'on est en voie de vivre l'expérience américaine: l'action en santé et sécurité du travail et les mécanismes nouveaux sont intégrés au cadre traditionnel de la négociation collective ou encore, peut-on supposer, là où les travailleurs ne sont pas syndiqués, au style habituel de gestion des ressources humaines.

\section{Joint Occupational Health and Safety Committees: Institutions of Cooperation or Negotiation?}

The purpose of this paper is to identify patterns in the behavior (or suggest hypotheses about the behavior) of the actors involved in joint committees on occupational health and safety. Specifically, the purpose is to see whether, in these committees, the parties adopt new cooperative attitudes on occupational health and safety, as promoted by Québec's law, or whether they still keep to their traditional adversarial attitudes on these issues.

In the case of unionized firms, it seems that employers do not recognize the legitimacy of labor's participation as a decision-maker on these issues; moreover, employers consider meetings of the joint committees as negotiation sessions and they tend to analyze the topics discussed at these meetings within the traditional framework of management rights and ability to pay. Union organizations also continue to see occupational health and safety as issues to be negotiated, issues on which gains can be made essentially through the bargaining process. This conclusion stems

29 Thomas KOCHAN, op. cit., p. 417. 
from the 3 following attitudes commonly held within union organizations: first, union representatives on these committees are integrated in the union structure: secondly, unions refuse to sit with non-unionized workers on these committees (in firms where not all workers are unionized); and, finally, in the study sessions they organize for their representatives on these committees, unions insist on the relationship between collective bargaining and what goes on in the committees.

In non-unionized firms, it can be suggested from a study of employer behavior on other issues that, unless external pressure is exerted, employers will do nothing to bring about the creation of joint committees on occupational health and safety; if a committee is created, management will consider it as subject to its own rights, not as an autonomous institution. As for the workers, they will adopt their "normal» behavior, the «passive» behavior they usually adopt towards their rights and prerogatives. They will not seek the creation of a joint committee, rather, they will tend, if need be, to call upon the governmental institution responsible for occupational health and safety. If a committee is created, it will not be an efficient means of worker participation.

Thus, it seems that, even if the mechanism is new, actors behave in the same traditional way. This corresponds in fact to the American experience, particularly the predisposition to integrate any new institution of cooperation in the firmly established collective bargaining process.

\section{Travail Labour Canada Canada}

\section{LES NORMES DU TRAVAIL AU CANADA}

\section{Édition de 1984}

Préparé par le service Analyse et recherche législatives de Travail Canada, cet ouvrage fait état des législations fédérale, provinciale et territoriale concernant l'âge de scolarité obligatoire et l'âge minimum d'admission à l'emploi, le salaire minimum, l'égalité de rémunération, la protection de la maternité, la durée du travail, le repos hebdomadaire, le congé annuel, les jours fériés payés et la cessation d'emploi.

N" de catalogue 1. 2-7. $1984 \mathrm{~F}$ ISBN 0-660-91259-7
Canada: $\$ 6.75$

à l'ètranger: $\$ 8.10$
$21.5 \times 28 \mathrm{~cm} 149 \mathrm{pp}$.

En vente au Canada par l'entremise des agents libraires agrés et autres librairies ou par la poste au

Centre d'édition du gouvernement du Canada

Approvisionnements et Services Canada

Ottawa, Ont. Canada. K1A OS9

Also available in English under the title Labour Standards in Canada, 1984 Edition. 